\title{
Intermolecular and Intramolecular $\alpha$-Amidoalkylation Reactions using Bismuth Triflate as Catalyst
}

by

Frédéric Pin, ${ }^{\dagger}$ Sébastien Comesse, ${ }^{\dagger}$ Bernard Garrigues, ${ }^{*}$ Štefan Marchalin ${ }^{*}$ and Adam Daïch ${ }^{*}$

URCOM, EA 3221, UFR des Sciences \& Techniques, Université du Havre, 25 rue Philippe Lebon, BP: 540, F-76058 Le Havre Cedex, France; Hétérochimie Fondamentale et Appliquée, UMR-5069 CNRS, Université Paul Sabatier, 118 route de Narbonne, F-31062 Toulouse Cedex 04, France; and

Department of Organic Chemistry, Slovak University of Technology, SK-81237 Bratislava, Slovak Republic

adam.daich@univ-lehavre.fr

RECEIVED DATE (to be automatically inserted after your manuscript is accepted if required according to the journal that you are submitting your paper to)

Bismuth(III) triflate mediated intermolecular and intramolecular $\alpha$-amidoalkylation processes.

Authors may by reached by phone at $(+33)$ 02-32-74-44-03, by fax at $(+33)$ 02-32-74-43-91 or by E.mail at the following address: adam.daich@univ-lehavre.fr

\section{$I^{\circ}$ - NMR discussion}

(Fore more details, please see NMR spectra of all products in the supporting Information file).

The structure of all products and intermediates reported herein was confirmed by their ${ }^{1} \mathrm{H},{ }^{13} \mathrm{C}$ NMR spectra including DEPT programs and elemental analyses. So, the ${ }^{1} \mathrm{H}$ NMR spectra of allyl derivatives $4 \mathbf{a}-\mathbf{I}$ and alkyl ones as $\mathbf{5 a}$ and $\mathbf{6 a}$ recorded in $\mathrm{CDCl}_{3}$ showed the methylene group of the $\mathrm{N}-\mathrm{CH}_{2}$ - functionalities as an $\mathrm{AB}$ system due to the diastereotopic effect with a coupling constant of $J=14.1-15.6 \mathrm{~Hz}$ characteristic of geminate protons. Also, the angular proton $\mathrm{CH}$ in $4 \mathbf{a}-\mathbf{i}, \mathbf{5 a}$ and $6 \mathbf{a}$ appears as a doublet of doublet centered at $\delta=3.53$ up to $5.07 \mathrm{ppm}$ with $J=3.9-5.0 \mathrm{~Hz}$ and $J=4.7-7.0$ $\mathrm{Hz}$ characteristic of the coupling observed in the $-\mathrm{CH}-\mathrm{CH}_{2}-$ enchainment installed in the above final allyl and alkyl products by the $\alpha$-amidoalkylation process. Of particular note, this proton reasoned in general up-field compared to the same one in corresponding $\alpha$-hydroxy lactams $2 \mathbf{a}-\mathbf{i}$ and $\alpha$-acetoxy lactams 3a-i in which it appeared as a doublet $(\mathrm{CH}(\mathrm{OH}))$ at $\delta=5.56$ up to $5.77 \mathrm{ppm}$ with $J=8.8-10.4$ $\mathrm{Hz}$ and as a singlet at $\delta=6.86$ up to $7.11 \mathrm{ppm}$, respectively. In the case of $\mathbf{3 i}$, this proton appears as 
doublet at $6.06 \mathrm{ppm}$ with $J=5.5 \mathrm{~Hz}$. More interestingly, the ${ }^{13} \mathrm{C}$ NMR spectra of 4a-i revealed the presence of two $\mathrm{sp}^{2}$-hybridized carbons corresponding to $\mathrm{CH}=\mathrm{CH}_{2}$ fragment of the allyl group and the absence of the quaternary ester carbonyl function $(\mathrm{C}=\mathrm{O})$ and $\mathrm{CH}_{3}$ of the acyl group, in the starting $\alpha$ acetoxy lactams 3a-i, respectively. These values are comparable to that reported in the literature for related allyl products. ${ }^{2}$ In the case of products $5 \mathbf{a}$ and $\mathbf{6 a}$, an additional acyl and benzoyl carbonyl function $(\mathrm{C}=\mathrm{O})$ instead of $-\mathrm{CH}=\mathrm{CH}_{2}$ residue were appeared at $\delta=205.8 \mathrm{ppm}$ and $\delta=197.3$, respectively.

Several key features in the ${ }^{1} \mathrm{H}$ and ${ }^{13} \mathrm{C}$ NMR spectra of the cyclized compounds $\mathbf{4 j} \mathbf{j} \mathbf{I}$ and $\mathbf{4 p}, \mathbf{g}$ were diagnostic of the structure. For instance, in the ${ }^{1} \mathrm{H}$ NMR spectra of isoindoloisoquioline $\mathbf{4} \mathbf{j}$ and isoindolo-[1,3]benzoxazines $\mathbf{4 k} \mathbf{k}, \mathbf{l}$ the methylene protons of the $-\mathrm{N}-\mathrm{CH}_{2}-$ functionalities, which were an $\mathrm{AB}$ system due to the diastereotopic effect in $\alpha$-acetoxy lactams congeners $\mathbf{3 j}$-1 (with $J=14.5,10.5$ and $11.5 \mathrm{~Hz}$, respectively), appeared also as an $\mathrm{AB}$ system but interestingly with increasing coupling constant values, which are respectively of $J=15.5,16.8$ and $17.0 \mathrm{~Hz} .^{1,2}$ The spectra showed also the disappearance of two signals corresponding to the $\mathrm{CH}_{3}$ protons of the lateral acetyl group and one aromatic proton in $\mathbf{3 j - 1}$. For pyrroloisoquinolinone $\mathbf{4 p}$, the ${ }^{1} \mathrm{H}$ NMR revealed the presence of the $\mathrm{CH}_{2}-\mathrm{CH}_{2}$ - group and absence of one proton in the aromatic region while in the NMR spectra of pyrroloisoquinolinone $\mathbf{4 g}$, this chain appears as two triplet at $\delta=2.94$ and 3.72 with $J=6.3 \mathrm{~Hz}$ as a consequence of the absence of the stereogenic center in the nitrogen adjacent position. They revealed also the presence of a singlet corresponding to the angular proton in $\mathbf{4 j - 1}$ at $\delta=5.69,5.96$ and 6.14 ppm, shifted downfield compared to those in corresponding $\alpha$-acetoxy lactams $\mathbf{3 j}$ ( $\delta=6.89 \mathrm{ppm}), \mathbf{3 k}$ $(\delta=7.18 \mathrm{ppm})$ and $\mathbf{3 l}(\delta=7.16 \mathrm{ppm})$, respectively. It is of particular note that the latter proton appeared as a doublet with trans coupling constant of $J=4.0 \mathrm{~Hz}$ in $(1 S, 10 \mathrm{bR})-1$-acetoxy-1,2,3,5,6,10bhexahydro-pyrrolo[2,1-a]isoquinolin-3-one $(\mathbf{4} \mathbf{p}),{ }^{3}$ while in the case of $\mathbf{4 q}$ no expected angular proton at nitrogen $\alpha$-position appeared. As a consequence of the spontaneous carbon-carbon double bond isomerization in the pyrrolidinone ring leading to the formation of the more stable isomer in which the olefin was in conjugation with the benzene ring, an angular proton at the adjacent position of the lactamic carbonyl function appeared as a doublet of quadruplet at $\delta=3.24 \mathrm{ppm}$ with $J=7.8 \mathrm{~Hz}(\mathrm{CH}$ $\mathrm{CH}_{3}$ coupling) and $\mathrm{J}=3.1 \mathrm{~Hz}(\mathrm{CH}-\mathrm{CH}=$ coupling $)$.

1 (a) Katritzky, A. R.; Mehta, S.; He, H.-Y. J. Org. Chem. 2001, 66, 148-152 and references therein. (b) Hucher, N.; Daïch, A.; Decroix, B. J. Heterocycl. Chem. 1998, 35, 1477-1483.

2 See Winn, M.; Zaugg, H. E. J. Org. Chem. 1968, 33, 3779 and also reference 1a.

3 Lee, Y. S.; Kang, D. W.; Lee, S-.J.; Park, H. J. Org. Chem. 1995, 60, 7149-7152. 
Carbon-13 NMR data proved to be of particular value in the assignment of structure of cyclized compounds as isoindoloisoquioline $\mathbf{4 j}$, isoindolo[1,3]benzoxazines $\mathbf{4 k}, \mathbf{l}$ and pyrrolo[2,1-a]isoquinolinones 4p,g. The most distinguishing feature in the ${ }^{13} \mathrm{C}$ NMR spectra was the regular appearance of one downfield signal at $\delta=168.2 \mathrm{ppm}, 168.2 \mathrm{ppm}$ and $168.6 \mathrm{ppm}$ for $\mathbf{4 j} \mathbf{j}, \mathbf{k}, \mathbf{l}$ and $\delta=170.3 \mathrm{ppm}$ and $180.0 \mathrm{ppm}$ for $\mathbf{4 p , g}$, which have been attributed to the lactamic carbonyl $\mathrm{C}=\mathrm{O}$. From these results, it is apparent that the variation of the central nucleus of the triheterocyclic systems $\mathbf{4 j} \mathbf{j} \mathbf{I}$ and $\mathbf{4 p}$ led to only small changes in the chemical shifts values for this resonance. In comparison with their $\alpha$ acetoxy lactams congeners $\mathbf{3 j} \mathbf{- 1}$ and $\mathbf{3 p}$, the difference of the chemical shift values are of $\Delta \delta=+0.2$, $+0.4,+0.8$ and $+0.2 \mathrm{ppm}$. But we noted in this data set for the same carbon in $\mathbf{4 g}$, a consistent downfield shift of $\Delta \delta=+9.2 \mathrm{ppm}$ in comparison between the cyclized product $\mathbf{4 g}$ and $\alpha$-acetoxy lactam 3g. This is due probably, in the latter case, to the presence of a double bond in conjugation with benzene ring as constraint system. Furthermore, the key feature in the ${ }^{13} \mathrm{C}$ NMR spectra of products $\mathbf{4 j - 1}, \mathbf{4 p}$ and $\mathbf{4 q}$ was the respective appearance of thirteen, seven and nine signals in the aromatic region. Moreover, two of these resonances including a carbonyl signal and one aromatic quaternary carbon disappear in the corresponding DEPT program spectra, as a consequence of the intramolecular $\alpha$-amidoalkylation cyclization. In the case of $\mathbf{4 q}$, an additional quaternary carbon located at $\alpha$-position of the lactamic nitrogen atom disappears also after similar NMR measurements.

\section{II- Experimental Section}

General remarks. Melting points were taken with a capillary melting point apparatus and are uncorrected. The infrared (IR) absorption spectra were determined as solutions in potassium bromide and are indicated in $\mathrm{cm}^{-1}$. The ${ }^{1} \mathrm{H}$ and ${ }^{13} \mathrm{C}$ NMR spectra were recorded as solutions in $\mathrm{CDCl}_{3}$ or DMSO- $d_{6}$ at 200 or $300 \mathrm{MHz}\left({ }^{1} \mathrm{H}\right)$ and 50.3 or $75 \mathrm{MHz}\left({ }^{13} \mathrm{C}\right)$, respectively, and chemical shifts $(\delta)$ are expressed in ppm relative to TMS as internal standard. Thin layer chromatography (TLC) was performed using of silica gel analytical plates $\left(F_{254}\right)$ of $0.25 \mathrm{~mm}$ thickness. The detection on TLC plates was performed by UV light at 254 or $365 \mathrm{~nm}$ or using $p$-anisaldehyde revelator. The analytical results of elemental analysis are within $0.4 \%$ of theoretical values and were obtained from INSA institution at Rouen, F-76130 Mt-St-Aignan in France.

6-(4-Methoxybenzyl)-6H-pyrrolo[3,4-b]pyridine-5,7-dione (1g). A mixture of $p$-methoxybenzylamine $(1.37 \mathrm{~g}, 10 \mathrm{mmol})$, pyridine-2,3-dicarboxylic anhydride $(1.49 \mathrm{~g}, 10 \mathrm{mmol})$ and three drops of anhydrous triethylamine in toluene $(100 \mathrm{~mL})$ was refluxed with a Dean-stark apparatus for 36 hours. The reaction mixture was cooled, then concentrated under reduced pressure. The residue was 
dissolved into dichloromethane $(20 \mathrm{~mL})$, washed with 5\% hydrochloric acid solution then with a 5\% sodium hydrogenocarbonate solution. The organic layer was dried over magnesium sulfate, concentrated under vacuo, and purified by chromatography on silica gel column using a mixture of cyclohexane/ethyl acetate (3/2) as eluent. This product was isolated as a white solid in $78 \%$ yield; $R_{f}$ 0.56 (cyclohexane/AcOEt 2:3); mp $130{ }^{\circ} \mathrm{C}$; IR (KBr): $v$ 1781, 1725, 1515, 1466, 1392, 1344, 1249 $\mathrm{cm}^{-1} ;{ }^{1} \mathrm{H}$ NMR $\left(\mathrm{CDCl}_{3}\right): \delta 3.74\left(\mathrm{~s}, 3 \mathrm{H}, \mathrm{OCH}_{3}\right), 4.82\left(\mathrm{~s}, 2 \mathrm{H}, \mathrm{CH}_{2} \mathrm{~N}\right), 6.83\left(\mathrm{~d}, 2 \mathrm{H}, J=8.6 \mathrm{~Hz}, \mathrm{H}_{\text {aro }}\right)$, $7.36\left(\mathrm{~d}, 2 \mathrm{H}, J=8.6 \mathrm{~Hz}, \mathrm{H}_{\mathrm{aro}}\right), 7.56\left(\mathrm{dd}, 1 \mathrm{H}, J=7.8\right.$ and $\left.5.5 \mathrm{~Hz}, \mathrm{H}_{\text {aro }}\right), 8.11(\mathrm{dd}, 1 \mathrm{H}, J=7.8$ and 1.6 $\left.\mathrm{Hz}, \mathrm{H}_{\text {aro }}\right), 8.91\left(\mathrm{dd}, 1 \mathrm{H}, J=5.5\right.$ and $\left.1.6 \mathrm{~Hz}, \mathrm{H}_{\text {aro }}\right) ;{ }^{13} \mathrm{C} \mathrm{NMR}\left(\mathrm{CDCl}_{3}\right): \delta 41.5\left(\mathrm{CH}_{2}\right), 55.4\left(\mathrm{CH}_{3}\right), 114.2$ $\left(2 \mathrm{CH}_{\text {aro }}\right), 127.5\left(\mathrm{CH}_{\text {aro }}\right), 127.6\left(\mathrm{C}_{\mathrm{q}}\right), 128.3\left(\mathrm{C}_{\mathrm{q}}\right), 129.6\left(\mathrm{C}_{\mathrm{q}}\right), 130.5\left(2 \mathrm{CH}_{\text {aro }}\right), 131.4\left(\mathrm{CH}_{\text {aro }}\right), 155.4$ $\left(\mathrm{CH}_{\text {aro }}\right), 159.5\left(\mathrm{C}_{\mathrm{q}}\right), 166.1(\mathrm{C}=\mathrm{O}), 166.2(\mathrm{C}=\mathrm{O})$; Anal. Calcd. for $\mathrm{C}_{15} \mathrm{H}_{12} \mathrm{~N}_{2} \mathrm{O}_{3}$ (268.08): $\mathrm{C}, 67.16 ; \mathrm{H}$, 4.51; N, 10.44. Found: C, 67.07; H, 4.42; N, 10.34.

2-(4-Methoxybenzyl)-2H-pyrrolo[3,4-b]quinoline-1,3-dione (1h). To a solution of quinoline-2,3dicarboxylic anhydride (995 mg, $5 \mathrm{mmol}$ ) in $50 \mathrm{~mL}$ of dry THF was added at room temperature under stirring dropwise $p$-methoxybenzylamine $(685 \mathrm{mg}, 5 \mathrm{mmol})$. After $2 \mathrm{~h}$ of reaction, 1 equiv. of DCC $(1.03 \mathrm{~g}, 5 \mathrm{mmol})$ and $10 \mathrm{~mol} \%$ of DMAP were added and the reaction was heated at reflux for $12 \mathrm{~h}$. The reaction mixture was could, filtered of on short column of a Celite pad and the filtrate we concentrated in vacuo. The residue was redissolved in dichloromethane and filtered again using same protocol. The solvent was carefully removed under vacuum to give white crystals, which were purified by flash-chromatography, eluting with cyclohexane/EtOAc 1:3. This led to isolation of the expected imide $\mathbf{1 h}$ as a white solid in $70 \%$ yield; $R_{f} 0.47$ (cyclohexane/AcOEt $1: 1$ ); mp $208{ }^{\circ} \mathrm{C}$; IR $(\mathrm{KBr}): v 1779,1720,1628,1615,1593,1515,1467,1395 \mathrm{~cm}^{-1} ;{ }^{1} \mathrm{H}$ NMR $\left(\mathrm{CDCl}_{3}\right): \delta 3.75(\mathrm{~s}, 3 \mathrm{H}$, $\left.\mathrm{OCH}_{3}\right), 4.91\left(\mathrm{~s}, 2 \mathrm{H}, \mathrm{CH}_{2} \mathrm{~N}\right), 6.83\left(\mathrm{~d}, 2 \mathrm{H}, J=8.6 \mathrm{~Hz}, \mathrm{H}_{\text {aro }}\right), 7.43\left(\mathrm{~d}, 2 \mathrm{H}, J=8.6 \mathrm{~Hz}, \mathrm{H}_{\text {aro }}\right), 7.73$ (dd, $1 \mathrm{H}, J=7.8$ and $7.0 \mathrm{~Hz}, \mathrm{H}_{\text {aro }}$ ), 7.91 (dd, $1 \mathrm{H}, J=8.6$ and $\left.7.0 \mathrm{~Hz}, \mathrm{H}_{\text {aro }}\right), 8.02\left(\mathrm{~d}, 1 \mathrm{H}, J=7.8 \mathrm{~Hz}, \mathrm{H}_{\text {aro }}\right.$ ), $8.40\left(\mathrm{~d}, 1 \mathrm{H}, J=8.6 \mathrm{~Hz}, \mathrm{H}_{\text {aro }}\right), 8.61\left(\mathrm{~s}, 1 \mathrm{H}, \mathrm{H}_{\text {aro }}\right) ;{ }^{13} \mathrm{C} \mathrm{NMR}\left(\mathrm{CDCl}_{3}\right): \delta 41.8\left(\mathrm{CH}_{2}\right), 55.5\left(\mathrm{CH}_{3}\right), 114.3$ $\left(2 \mathrm{CH}_{\text {aro }}\right), 123.2\left(\mathrm{C}_{\mathrm{q}}\right), 128.3\left(\mathrm{C}_{\mathrm{q}}\right), 128.9\left(\mathrm{C}_{\mathrm{q}}\right), 129.7\left(\mathrm{CH}_{\text {aro }}\right), 130.1\left(\mathrm{CH}_{\text {aro }}\right), 130.6\left(2 \mathrm{CH}_{\text {aro }}\right), 131.7$ $\left(\mathrm{CH}_{\text {aro }}\right), 132.8\left(\mathrm{CH}_{\text {aro }}\right), 132.9\left(\mathrm{CH}_{\text {aro }}\right), 150.8\left(\mathrm{C}_{\mathrm{q}}\right), 151.0\left(\mathrm{C}_{\mathrm{q}}\right), 159.6\left(\mathrm{C}_{\mathrm{q}}\right), 165.9(\mathrm{C}=\mathrm{O}), 166.1(\mathrm{C}=\mathrm{O})$; Anal. Calcd. for $\mathrm{C}_{19} \mathrm{H}_{14} \mathrm{~N}_{2} \mathrm{O}_{3}$ (318,1): C, 71.69; H, 4.43; N, 8.80. Found: C, 71.54; H, 4.34; N, 8.71.

General Procedure for the Reduction of Imides 1a-p. $\mathrm{NaBH}_{4}$ (3 to 6 equiv.) was added by portions over 20 minutes at $0{ }^{\circ} \mathrm{C}$ to a stirred solution of the imide 1a-q (1 equiv.) in $\mathrm{MeOH}(20 \mathrm{~mL}$ per $1 \mathrm{mmol}$ of imide $\mathbf{1}$ ). The reaction mixture was directly concentrated under reduce pressure, then $\mathrm{CH}_{2} \mathrm{Cl}_{2}$ was added. The organic layer was washed with water, brine, dried over $\mathrm{MgSO}_{4}$ and the solvent evaporated. The residue was then purified by chromatography on a silica gel column to give 
the expected hydroxy lactams 2a-q. For reduction of imides $\mathbf{1 g}, \mathbf{h}, 1.2$ equiv. $\mathrm{NaBH}_{4}$ and 1 equiv. of $\mathrm{Mg}\left(\mathrm{ClO}_{4}\right)_{2}$ in a mixture of $\mathrm{THF} / \mathrm{CHCl}_{3}(1 / 1$ of $\mathrm{V} / \mathrm{V})$ were used.

2-(Furan-2-ylmethyl)-3-hydroxyisoindolin-1-one (2a). This product was isolated as a brown

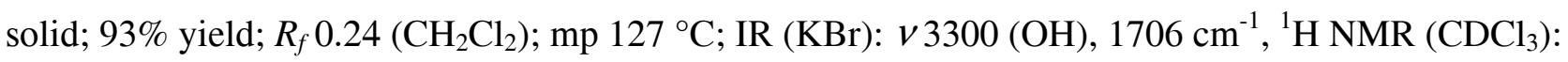
$\delta 3.90(\mathrm{~d}, 1 \mathrm{H}, J=9.4 \mathrm{~Hz}, \mathrm{OH}), 4.24\left(\mathrm{~d}, 1 \mathrm{H}, J=15.6 \mathrm{~Hz}, \mathrm{CH}_{2} \mathrm{~N}\right), 4.60\left(\mathrm{~d}, 1 \mathrm{H}, J=15.6 \mathrm{~Hz}, \mathrm{CH}_{2} \mathrm{~N}\right)$, $5.69(\mathrm{~d}, 1 \mathrm{H}, J=9.4 \mathrm{~Hz}, \mathrm{CH}), 6.25\left(\mathrm{~s}, 2 \mathrm{H}, \mathrm{H}_{\text {fur }}\right), 7.28\left(\mathrm{~s}, 1 \mathrm{H}, \mathrm{H}_{\text {fur }}\right), 7.38-7.64\left(\mathrm{~m}, 4 \mathrm{H}, \mathrm{H}_{\text {aro }}\right) ;{ }^{13} \mathrm{C} \mathrm{NMR}$ $\left(\mathrm{CDCl}_{3}\right): \delta 35.6\left(\mathrm{CH}_{2}\right), 81.6(\mathrm{CH}), 108.7\left(\mathrm{CH}_{\text {aro }}\right), 110.6\left(\mathrm{CH}_{\text {aro }}\right), 123.6\left(\mathrm{CH}_{\text {aro }}\right), 123.7\left(\mathrm{CH}_{\text {aro }}\right), 129.9$ $\left(\mathrm{CH}_{\text {aro }}\right), 131.3\left(\mathrm{C}_{\mathrm{q}}\right), 132.7\left(\mathrm{CH}_{\text {aro }}\right), 142.7\left(\mathrm{CH}_{\text {aro }}\right), 144.1\left(\mathrm{C}_{\mathrm{q}}\right), 150.3\left(\mathrm{C}_{\mathrm{q}}\right), 167.4(\mathrm{C}=\mathrm{O})$; Anal. Calcd. for $\mathrm{C}_{13} \mathrm{H}_{11} \mathrm{NO}_{3}$ (229.07): C, 68.11; H, 4.84; N, 6.11. Found: C, 68.01; H, 4.76; N, 6.05.

3-Hydroxy-2-(4-methoxybenzyl)-isoindolin-1-one (2f). This product was isolated as a white solid; 94\% yield; $R_{f} 0.33$ (cyclohexane/AcOEt 1:1); mp $158{ }^{\circ} \mathrm{C}$; IR (KBr): $v 3691,3559,2686,2411$, 1700, 1684, $1613 \mathrm{~cm}^{-1} ;{ }^{1} \mathrm{H}$ NMR $\left(\mathrm{CDCl}_{3}\right): \delta 3.35(\mathrm{~d}, 1 \mathrm{H}, J=10.4 \mathrm{~Hz}, \mathrm{OH}), 3.74\left(\mathrm{~s}, 3 \mathrm{H}, \mathrm{OCH}_{3}\right), 4.17$ $\left(\mathrm{d}, 1 \mathrm{H}, J=14.9 \mathrm{~Hz}, \mathrm{CH}_{2} \mathrm{~N}\right), 4.79\left(\mathrm{~d}, 1 \mathrm{H}, J=14.9 \mathrm{~Hz}, \mathrm{CH}_{2} \mathrm{~N}\right), 5.56(\mathrm{~d}, 1 \mathrm{H}, J=10.4 \mathrm{~Hz}, \mathrm{CH}), 6.79$ (d, $\left.2 \mathrm{H}, J=8.6 \mathrm{~Hz}, \mathrm{H}_{\text {aro }}\right), 7.22\left(\mathrm{~d}, 2 \mathrm{H}, J=8.6 \mathrm{~Hz}, \mathrm{H}_{\text {aro }}\right), 7.38-7.55\left(\mathrm{~m}, 3 \mathrm{H}, \mathrm{H}_{\text {aro }}\right), 7.63(\mathrm{~d}, 1 \mathrm{H}, J=7.0 \mathrm{~Hz}$, $\left.\mathrm{H}_{\text {aro }}\right) ;{ }^{13} \mathrm{C}$ NMR $\left(\mathrm{CDCl}_{3}\right): \delta 42.2\left(\mathrm{CH}_{2}\right), 55.4\left(\mathrm{CH}_{3}\right), 81.1(\mathrm{CH}), 114.2\left(2 \mathrm{CH}_{\text {aro }}\right), 123.5\left(\mathrm{CH}_{\text {aro }}\right), 123.6$ $\left(\mathrm{CH}_{\text {aro }}\right), 129.1\left(\mathrm{C}_{\mathrm{q}}\right), 129.8\left(\mathrm{CH}_{\text {aro }}\right), 130.0\left(2 \mathrm{CH}_{\text {aro }}\right), 131.5\left(\mathrm{C}_{\mathrm{q}}\right), 132.5\left(\mathrm{CH}_{\text {aro }}\right), 144.2\left(\mathrm{C}_{\mathrm{q}}\right), 159.2\left(\mathrm{C}_{\mathrm{q}}\right)$, 167.5 (C=O); Anal. Calcd. for $\mathrm{C}_{16} \mathrm{H}_{15} \mathrm{NO}_{3}$ (269.11): C, 71.36; H, 5.61; N, 5.20. Found: C, 71.22; H, $5.49 ; \mathrm{N}, 5.15$.

7-Hydroxy-6,7-dihydro-6-(4-methoxybenzyl)-pyrrolo[3,4-b]pyridin-5-one (2g). This product was isolated as a white solid; $72 \%$ yield; $R_{f} 0.27$ (cyclohexane/AcOEt 1:4); mp $170{ }^{\circ} \mathrm{C}$; IR (KBr): $v$ $3164,1663,1615,1587,1513,1439,1422 \mathrm{~cm}^{-1} ;{ }^{1} \mathrm{H}$ NMR (DMSO-d $): \delta 3.76$ (s, 3H, $\mathrm{OCH}_{3}$ ), $4.34(\mathrm{~d}$, $\left.1 \mathrm{H}, J=14.9 \mathrm{~Hz}, \mathrm{CH}_{2} \mathrm{~N}\right), 4.91\left(\mathrm{~d}, 1 \mathrm{H}, J=14.9 \mathrm{~Hz}, \mathrm{CH}_{2} \mathrm{~N}\right), 5.62(\mathrm{~d}, 1 \mathrm{H}, J=8.8 \mathrm{~Hz}, \mathrm{CH}), 6.91(\mathrm{~d}, 2 \mathrm{H}$, $\left.J=8.8 \mathrm{~Hz}, \mathrm{H}_{\text {aro }}\right), 6.99(\mathrm{~d}, 1 \mathrm{H}, J=8.8 \mathrm{~Hz}, \mathrm{OH}), 7.29$ (d, 2H, $J=8.6 \mathrm{~Hz}, \mathrm{H}_{\text {aro }}$ ), 7.59 (dd, 1H, $J=7.8$ and $\left.4.4 \mathrm{~Hz}, \mathrm{H}_{\text {aro }}\right), 8.13\left(\mathrm{dd}, 1 \mathrm{H}, J=7.8\right.$ and $\left.1.5 \mathrm{~Hz}, \mathrm{H}_{\text {aro }}\right), 8.80\left(\mathrm{dd}, 1 \mathrm{H}, J=4.4\right.$ and $\left.1.5 \mathrm{~Hz}, \mathrm{H}_{\text {aro }}\right) ;{ }^{13} \mathrm{C}$ NMR (DMSO-d $\left.d_{6}\right): \delta 41.5\left(\mathrm{CH}_{2}\right), 55.1\left(\mathrm{CH}_{3}\right), 80.4(\mathrm{CH}), 113.9\left(2 \mathrm{CH}_{\text {aro }}\right), 124.6\left(\mathrm{CH}_{\text {aro }}\right), 125.2\left(\mathrm{C}_{\mathrm{q}}\right)$, $129.2\left(2 \mathrm{CH}_{\text {aro }}\right), 129.3\left(\mathrm{C}_{\mathrm{q}}\right), 131.1\left(\mathrm{CH}_{\text {aro }}\right), 152.8\left(\mathrm{CH}_{\text {aro }}\right), 158.5\left(\mathrm{C}_{\mathrm{q}}\right), 163.8\left(\mathrm{C}_{\mathrm{q}}\right), 164.5(\mathrm{C}=\mathrm{O})$; Anal. Calcd. for $\mathrm{C}_{15} \mathrm{H}_{14} \mathrm{~N}_{2} \mathrm{O}_{3}(270,1)$ : C, 66.66; H, 5.22; N, 10.36. Found: C, 66.53; H, 5.09; N, 10.28.

3-Hydroxy-2,3-dihydro-2-(4-methoxybenzyl)-pyrrolo[3,4-b]quinolin-1-one (2h). This product was isolated as a white solid; $77 \%$ yield; $R_{f} 0.47$ (cyclohexane/AcOEt 2:3); mp $211{ }^{\circ} \mathrm{C}$; IR (KBr): $v$ 3066, 1706, 1634, 1616, 1514, 1428, $1412 \mathrm{~cm}^{-1}$; ${ }^{1} \mathrm{H}$ NMR (DMSO- $\left.d_{6}\right): \delta 3.78$ (s, 3H, $\mathrm{OCH}_{3}$ ), $4.43(\mathrm{~d}$, $\left.1 \mathrm{H}, J=15.1 \mathrm{~Hz}, \mathrm{CH}_{2} \mathrm{~N}\right), 4.98\left(\mathrm{~d}, 1 \mathrm{H}, J=15.1 \mathrm{~Hz}, \mathrm{CH}_{2} \mathrm{~N}\right), 5.77(\mathrm{~d}, 1 \mathrm{H}, J=9.0 \mathrm{~Hz}, \mathrm{CH}), 6.94(\mathrm{~d}, 2 \mathrm{H}$, $\left.J=8.4 \mathrm{~Hz}, \mathrm{H}_{\text {aro }}\right), 7.08(\mathrm{~d}, 1 \mathrm{H}, J=9.0 \mathrm{~Hz}, \mathrm{OH}), 7.35\left(\mathrm{~d}, 2 \mathrm{H}, J=8.4 \mathrm{~Hz}, \mathrm{H}_{\text {aro }}\right), 7.76(\mathrm{dd}, 1 \mathrm{H}, J=7.8$ 
and $\left.7.0 \mathrm{~Hz}, \mathrm{H}_{\text {aro }}\right), 7.95\left(\mathrm{dd}, 1 \mathrm{H}, J=8.4\right.$ and $\left.7.0 \mathrm{~Hz}, \mathrm{H}_{\text {aro }}\right), 8.19\left(\mathrm{~d}, 1 \mathrm{H}, J=7.8 \mathrm{~Hz}, \mathrm{H}_{\text {aro }}\right), 8.26(\mathrm{~d}, 1 \mathrm{H}, J$ $\left.=8.4 \mathrm{~Hz}, \mathrm{H}_{\text {aro }}\right), 8.82\left(\mathrm{~s}, 1 \mathrm{H}, \mathrm{H}_{\mathrm{aro}}\right) ;{ }^{13} \mathrm{C} \mathrm{NMR}\left(\mathrm{DMSO}-d_{6}\right): \delta 41.7\left(\mathrm{CH}_{2}\right), 55.0\left(\mathrm{CH}_{3}\right), 80.6(\mathrm{CH}), 113.9$ $\left(2 \mathrm{CH}_{\text {aro }}\right), 123.0\left(\mathrm{C}_{\mathrm{q}}\right), 127.3\left(\mathrm{CH}_{\text {aro }}\right), 127.7\left(\mathrm{C}_{\mathrm{q}}\right), 129.0\left(\mathrm{CH}_{\text {aro }}\right), 129.1\left(\mathrm{C}_{\mathrm{q}}\right), 129.2\left(2 \mathrm{CH}_{\text {aro }}\right), 129.8$ $\left(\mathrm{CH}_{\text {aro }}\right), 131.5\left(\mathrm{CH}_{\text {aro }}\right), 131.9\left(\mathrm{CH}_{\text {aro }}\right), 149.2\left(\mathrm{C}_{\mathrm{q}}\right), 158.5\left(\mathrm{C}_{\mathrm{q}}\right), 162.5\left(\mathrm{C}_{\mathrm{q}}\right), 164.3(\mathrm{C}=\mathrm{O})$; Anal. Calcd. for $\mathrm{C}_{19} \mathrm{H}_{16} \mathrm{~N}_{2} \mathrm{O}_{3}$ (320.12): C, 71.24; H, 5.03; N, 8.74. Found: C, 71.07; H, 5.00; N, 8.66.

5-Hydroxy-3-methyl-1-phenethyl-1H-pyrrol-2(5H)-one (2qB). To a solution of 3-methyl-1phenethyl-1H-pyrrole-2,5-dione (1q, $1.075 \mathrm{~g}, 5 \mathrm{mmol})$ in methanol $20 \mathrm{~mL}$ was added in one portion and under stirring cerium(III) chloride heptahydrate $(1.86 \mathrm{~g}, 5 \mathrm{mmol})$. After $10 \mathrm{~min}$ of reaction at room temperature the solution was cooled to $0{ }^{\circ} \mathrm{C}$ and $190 \mathrm{mg}$ of sodium borohydride $(5 \mathrm{mmol})$ was added portionwise. After $1 \mathrm{~h}$ of reaction at $0{ }^{\circ} \mathrm{C}$ and additional $1 \mathrm{~h}$ at room temperature, the reaction mixture was quenched with ice water and methanol removed under reduced pressure. The solution was extracted twice with dichloromethane $(10 \mathrm{~mL})$ and the combined organic layers washed with water, brine, dried over $\mathrm{MgSO}_{4}$ and evaporated to dryness to give after chromatography separation, eluting with cyclohexane/AcOEt 1:4), the expected minor hydroxy lactam 2 qB in $28.7 \%$ yield (overall yield of $86 \%$ ). This product was isolated as a white solid; $R_{f} 0.25$ (cyclohexane/AcOEt 1:4); IR (KBr): $v 3054,1712,1645,1612,1507,1433 \mathrm{~cm}^{-1} ;{ }^{1} \mathrm{H}$ NMR $\left(\mathrm{CDCl}_{3}\right): \delta 1.85\left(\mathrm{~s}, 3 \mathrm{H}, \mathrm{CH}_{3}\right), 2.13$ (d, $1 \mathrm{H}, J=11.3 \mathrm{~Hz}, \mathrm{OH}), 2.88\left(\mathrm{dd}, 2 \mathrm{H}, J=7.8\right.$ and $7.0 \mathrm{~Hz}, \mathrm{CH}_{2}$ ), 3.51 (ddd, $1 \mathrm{H}, J=14.9,7.8$ and 7.0 $\left.\mathrm{Hz}, \mathrm{NCH}_{2}\right), 3.76$ (ddd, $1 \mathrm{H}, J=14.9,7.8$ and $\left.7.0 \mathrm{~Hz}, \mathrm{NCH}_{2}\right), 4.98(\mathrm{~d}, 1 \mathrm{H}, J=11.3 \mathrm{~Hz}, \mathrm{CHOH}), 6.45$ $(\mathrm{s}, 1 \mathrm{H},=\mathrm{CH}), 7.16-7.31\left(\mathrm{~m}, 5 \mathrm{H}, \mathrm{H}_{\mathrm{aro}}\right) ;{ }^{13} \mathrm{C} \mathrm{NMR}\left(\mathrm{CDCl}_{3}\right): \delta 11.1\left(\mathrm{CH}_{3}\right), 34.9\left(\mathrm{CH}_{2}\right), 41.2\left(\mathrm{CH}_{2}\right), 82.2$ $(\mathrm{CH}), 126.7\left(\mathrm{CH}_{\text {aro }}\right), 128.8\left(2 \mathrm{CH}_{\text {aro }}\right), 129.0\left(2 \mathrm{CH}_{\text {aro }}\right), 137.1\left(\mathrm{C}_{\mathrm{q}}\right), 138.6\left(\mathrm{CH}_{\text {aro }}\right), 139.1\left(\mathrm{C}_{\mathrm{q}}\right), 170.6$ $(\mathrm{C}=\mathrm{O})$; Anal. Calcd. for $\mathrm{C}_{13} \mathrm{H}_{15} \mathrm{NO}_{2}$ (217.11): C, 71.87; H, 6.96; N, 6.45. Found: C, 71.69; H, 6.87; N, 6.37 . 